\title{
Extracellular Matrix Scaffolds Are Repopulated by Bone Marrow-Derived Cells in a Mouse Model of Achilles Tendon Reconstruction
}

\author{
Thore Zantop, ${ }^{1}$ Thomas W. Gilbert, ${ }^{2}$ Mervin C. Yoder, ${ }^{3}$ Stephen F. Badylak ${ }^{2}$ \\ ${ }^{1}$ Department of Trauma, Hand and Reconstructive Surgery, Wilhelms University, Muenster, Germany \\ ${ }^{2}$ McGowan Institute for Regenerative Medicine, University of Pittsburgh, 100 Technology Drive, \\ Suite 200, Pittsburgh, Pennsylvania 15219 \\ ${ }^{3}$ Herman B. Wells Center for Pediatric Research, Indiana University School of Medicine, Indianapolis, Indiana
}

Received 24 May 2004; accepted 3 August 2005

Published online 28 April 2006 in Wiley InterScience (www.interscience.wiley.com). DOI 10.1002/jor.20071

\begin{abstract}
The extracellular matrix derived from porcine small intestinal submucosa (SIS-ECM), an FDA-approved material currently used clinically for rotator cuff repair, has been shown to attract bone marrow-derived cells during in vivo remodeling of a subcutaneous implant and produce chemoattractant peptides following chemical degradation in vitro. The purpose of the present study was to determine if bone marrow-derived cells participate in the long-term remodeling of the Achilles tendon in a mouse model when repaired with SIS-ECM. A 2-mm gap was produced in the Achilles tendon of 40 chimeric mice produced to express green fluorescent protein (GFP) in all of their bone marrow-derived cells. Tendons were repaired by replacing the resected section with autologous tendon tissue or with a single layer sheet of lyophilized SIS-ECM. Four animals from each treatment group were sacrificed at $1,2,4,8$, and 16 weeks, and sections were harvested for histologic and fluorescence microscopy. Both groups showed accumulation of GFP-expressing marrow-derived cells at the site of tendon remodeling at 1 and 2 weeks that were associated with areas of angiogenesis and inflammation. By 16 weeks, the SIS-ECM-treated group showed GFP expressing cells throughout the remodeled tendon in the absence of any inflammatory response, while the autologous tendon repair group showed no GFP expressing cells within the tendon except for occasional cells in the lumen of blood vessels. An SIS-ECM scaffold used for tendon repair recruits a population of bone marrow-derived cells that participates in the long-term remodeling process. The ability of SIS-ECM to recruit a population of marrow-derived cells to the remodeling site may alter the default mechanism of tendon healing. The involvement of these cells in the remodeling process may explain in part the process of site specific constructive remodeling as opposed to scar tissue formation when the ECM is used as a biologic scaffold for tendon reconstruction. (c) 2006 Orthopaedic Research Society. Published by Wiley Periodicals, Inc. J Orthop Res 24:1299-1309, 2006
\end{abstract}

Keywords: bone marrow-derived cells; extracellular matrix; Achilles tendon repair; injury; small intestinal submucosa

\section{INTRODUCTION}

Biologic scaffolds derived from extracellular matrix (ECM) have been used for repair of musculotendinous tissues, including the Achilles tendon and rotator cuff. ${ }^{1-3}$ Such scaffolds include Restore $^{\mathrm{TM}}$, CuffPatch ${ }^{\mathrm{TM}}$, GraftJacket ${ }^{\mathrm{R}}$, Tissue-

Correspondence to: Stephen F. Badylak (Telephone: 412235-5144; Fax: 412-235-5110; E-mail: badylaks@upmc.edu)

๑) 2006 Orthopaedic Research Society. Published by Wiley Periodicals, Inc.
Mend $^{\mathbb{R}}$, and Permacol ${ }^{\mathrm{TM}}$. Porcine small intestinal submucosa (SIS-ECM), the material from which Restore $^{\mathrm{TM}}$ is manufactured, is one ECM scaffold that has been investigated extensively in preclinical studies for a variety of clinical applications. In general, the host tissue response to SISECM has shown constructive remodeling at the site of the scaffold placement with functional tissue that is grossly and histologically similar in appearance to the native tissue. ${ }^{4-11}$ The SIS-ECM scaffold is rapidly resorbed, with approximately 
$40-60 \%$ of the ECM scaffold mass replaced by new host ECM within the first 4 weeks and complete replacement by 3 months after surgery. ${ }^{12}$

The mechanisms by which the remodeling process occurs are not fully understood. SIS-ECM has been found to contain a number of growth factors including transforming growth factor- $\beta 1$, basic-fibroblast growth factor, and vascular endothelial growth factor, ${ }^{13,14}$ and these growth factors retain most of their biological activity through the processing of the scaffold for clinical use. ${ }^{15,16} \mathrm{It}$ is likely that the presence of these growth factors contributes to migration of cells into the scaffold and behavior of these cells once they arrive. The scaffold also serves to transmit mechanical signals to host cells that populate the material, and these signals are, in part, a function of the rehabilitation protocol followed by the patient.

It is generally accepted that following tendon injury, the healing is characterized by an infiltration of inflammatory cells that shift from primarily neutrophils during the acute phase (e.g., first 48$72 \mathrm{~h}$ ) to a primarily mononuclear macrophage population thereafter. In the absence of complicating factors such as infection, the inflammatory cells become less abundant within 10-14 days as fibroblasts migrate into the remodeling tendon from the adjacent tissue. ${ }^{17-19}$ However, the cells that populate the SIS-ECM scaffold after surgical implantation are almost exclusively mononuclear in morphology after day 3 , and these mononuclear cells progressively increase in number for at least 14-28 days. This unusual cellular response is associated with new ECM deposition by the host and a site-specific constructive remodeling process. In a previous study in which SIS-ECM was implanted subcutaneously, ${ }^{20}$ it was shown that a portion of the cells that participated in the remodeling of SIS-ECM were bone marrow-derived cells, and they participated in the remodeling beyond the immediate postsurgical inflammatory response. The objective of the present study was to determine if bone marrow-derived cells participate in the long-term remodeling of a musculotendinous tissue when SIS-ECM is used in a mouse model of Achilles tendon repair.

\section{MATERIALS AND METHODS}

\section{SIS-ECM Preparation}

The porcine SIS-ECM was prepared as described previously. ${ }^{21}$ Briefly, the small intestine was mechanically delaminated to remove the tunica muscularis externa and the majority of the tunica mucosa. The remaining tunica submucosa, muscularis mucosa, and basilar portion of the lamina propria was then disinfected and decellularized in a $0.1 \%$ peracetic acid solution followed by two rinses each in phosphate-buffered saline and deionized water. This process yielded an acellular material, which was then lyophilized and terminally sterilized with ethylene oxide. A single layer form of SIS-ECM was used such that the long axis of the device corresponded with the direction of preferred fiber alignment of SIS-ECM. ${ }^{22}$

\section{Chimeric Mouse Model}

Six- to 8-week-old C57BL/6J mice were lethally irradiated with $11 \mathrm{~Gy}$ of gamma irradiation (96 cGy/min) administered in two doses divided by $4 \mathrm{~h}$ using a ${ }^{137} \mathrm{Cs}$ irradiator (Nordion, Kanata, Canada). The lethally irradiated C57BL/6J mice were transplanted via tail injection with $5 \times 10^{6}$ low-density mononuclear cells isolated from B6.ACTbEGFP mice that express green fluorescence protein (GFP) in all of their cells. Recipient animals were tested at monthly intervals to assay for the level of donor cell chimerism via sampling of peripheral blood cells. Stable chimerism posttransplant was achieved in 4 months. When the blood of the host animals was $100 \%$ donor origin (GFP positive), the mice were considered ready for use in the scaffold implantation study. ${ }^{23}$

\section{Study Groups and Surgical Technique}

All procedures were performed as approved by the Institutional Animal Care and Use Committee at the University of Pittsburgh (Protocol \#0405959) and the animal care complied with the NIH Guidelines for the Care and Use of Laboratory Animals.

Forty chimeric mice that expressed GFP in all of their bone marrow-derived cells were anesthetized with inhalant Isofluorane using a nose cone. The right hind limb of each animal was shaved and prepared for surgery using sterile technique. Under magnification, the Achilles tendon was exposed through an anterolateral skin incision and the surrounding paratenon incised. Two 7-0 Vicryl sutures (Ethicon, Somerville, NJ) were placed proximal and distal in the Achilles tendon. The spacing of the two sutures was $3 \mathrm{~mm}$. In group $1(n=20)$, the proximal and distal suture were stitched through the intervening tendon tissue creating two loops of suture (Fig. 1A). The tendon was then transected within each loop, creating the autologous tendon graft. The two suture loops were pulled tight and knotted to secure the graft. In group $2(n=20)$, a 2 -mm segment of the tendon was resected and a $3 \times 2 \mathrm{~mm}$ single-layer sheet of lyophilized SIS-ECM was attached to the free ends of the tendon via the two placed sutures (Fig. 1B). Prior to closing the skin, the graft was rehydrated with sterile saline, and the graft was tubularized around the free 
A
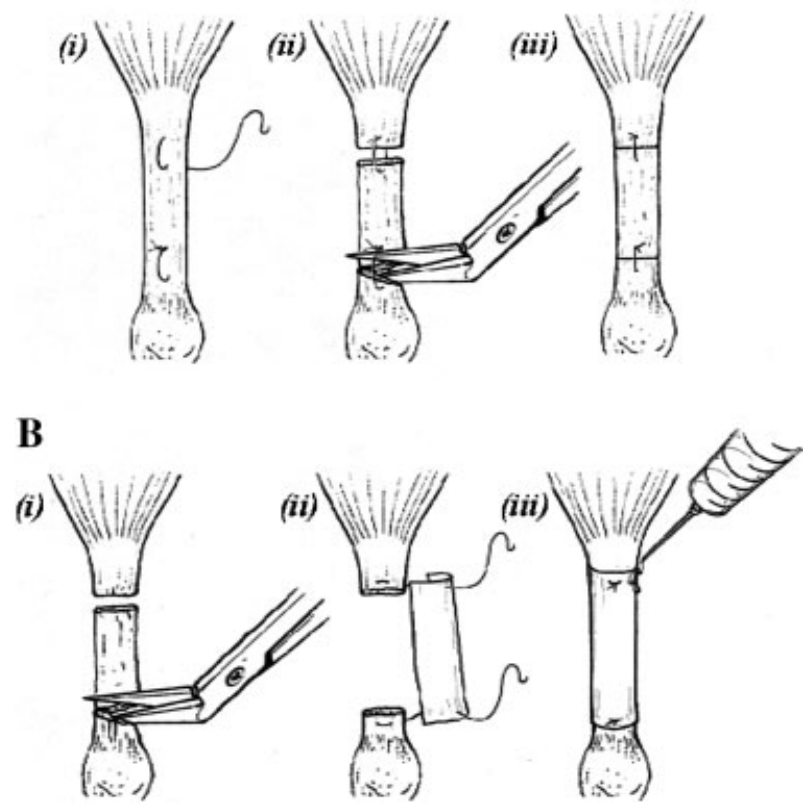

Figure 1. (A) The control procedure was performed by first (i) placing 7-0 prolene suture loops $2 \mathrm{~cm}$ apart in the midsubstance of the tendon. The tendon was then (ii) cut within the suture loops to hold the explanted tendon in place. The sutures were finally (iii) pulled taught and knotted to secure the autologous tendon graft. (B) The SIS repair procedure was performed by first (i) cutting a 2 -cm length from the midsubstance of the tendon. (ii) A single layer of lyophilized SIS-ECM was then secured to the cut ends of the tendon with 7-0 prolene suture. Finally, the sutures were (iii) pulled taught and knotted to secure the SIS-ECM graft and the graft was hydrated with saline.

ends of the tendon (Fig. 1B). In both groups the wound was closed in layers using 7-0 Vicryl sutures. Remodeling was evaluated in four animals in each group at time points of $1,2,4,8$, and 16 weeks after surgery.

\section{Specimen Collection and Analysis}

After sacrifice, the experimental and contralateral Achilles tendons were harvested from each animal and fixed in $4 \%$ paraformaldehyde. The tissue was then embedded in paraffin and $5-\mu \mathrm{m}$ sections were obtained. One unstained section was used to visualize the GFP protein, and adjacent sections were processed for staining with H\&E or Masson's Trichrome. The unprocessed section was then examined with fluorescent microscopy for the presence of green fluorescing cells, and photomicrographs were obtained at $20 \times$. A photomicrograph was also obtained for the same area of the tendon from adjacent tissue sections that were stained with H\&E or Masson's Trichrome to assess the histological appearance in the vicinity of the cells expressing GFP.

\section{RESULTS}

All animals tolerated the surgical procedure without adverse events and all animals were ambulatory immediately after surgery without apparent lameness.

\section{Native Achilles Tendon}

Histologically, the native Achilles tendon was composed of a highly organized collagenous matrix. Scattered spindle-shaped fibroblasts could be seen between highly aligned collagen fibers. In the paratendinous tissue, vascular structures were aligned longitudinal to the tendon. No GFP expressing marrow-derived cells were found in the intact Achilles tendon.

\section{Autologous Achilles Tendon Graft}

At necropsy, all tendon repairs remained intact. Gross examination showed that the reconstructed tendon of both groups had a slightly increased diameter when compared to the intact contralateral tendon at all time points. One week after surgery, the autologous tendon implant was difficult to identify because of the presence of dense granulation tissue. Histological examination at 1 and 2 weeks after surgery showed a dense infiltration of mononuclear cells and abundant new blood vessels (Fig. 2A). A robust accumulation of GFP-expressing marrow-derived cells was observed at the site of remodeling, generally associated with areas of angiogenesis and inflammation (Fig. 2B). By 4 weeks after surgery, the remodeled tendon consisted of organized collagenous tissue, and the cellularity had diminished to near normal. At 4, 8, and 16 weeks, no GFP expressing marrow-derived cells were observed in the tendon body, but occasional green fluorescent cells could be identified around or within vascular structures of the paratendinous tissue. Representative photomicrographs of the autologous repair group 4 weeks after surgery are shown in Figure $3 \mathrm{~A}$ and B. Figure 4 shows the histologic appearance of remodeled tissue 16 weeks after surgery. Table 1 provides a semiquantitative summary of the inflammatory cells, bone marrow-derived cells, mononuclear cells, and collagenous tissue of the autologous tendon repair and SIS-ECM repair groups compared to normal tendon at each time point. 
A

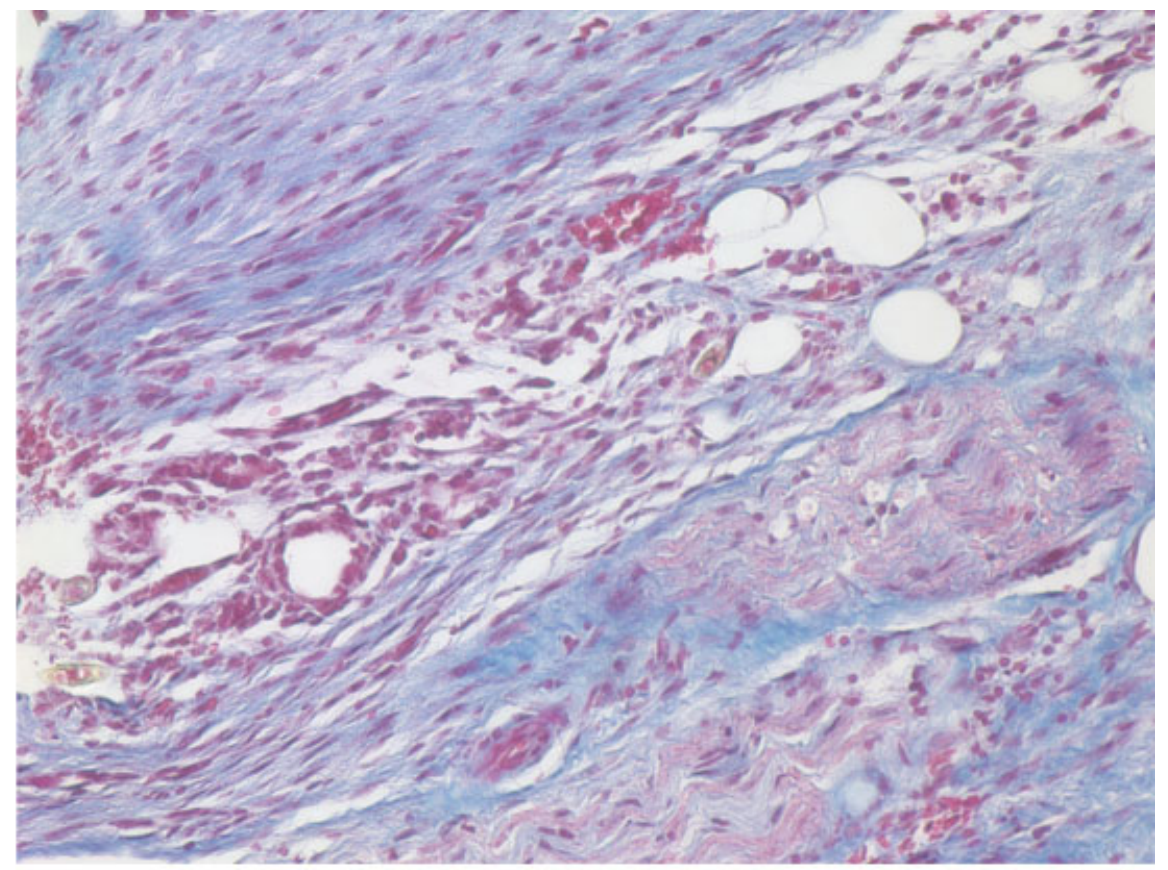

B

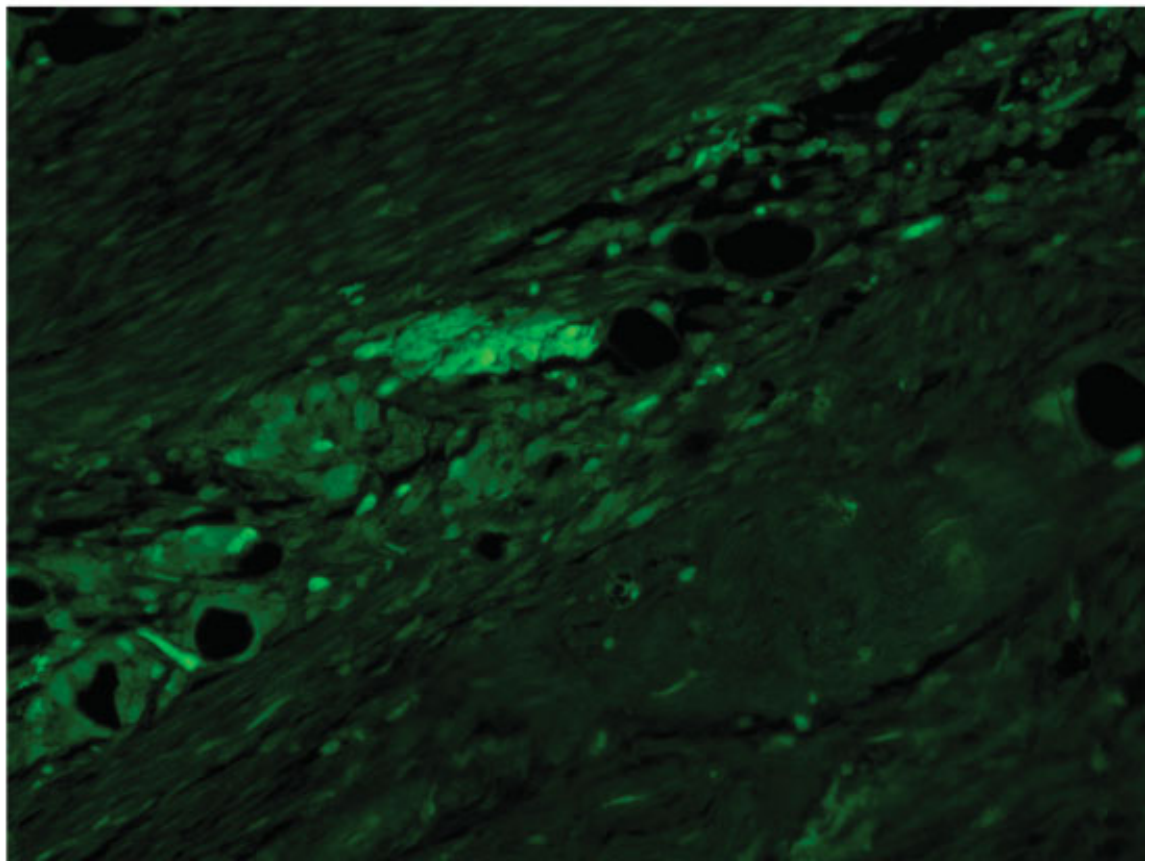

Figure 2. (A) At 2 weeks after surgery, the autologous tendon repair group showed a dense mononuclear cell infiltration, abundant blood vessels, and acute inflammation (Masson's Trichrome 20×). (B) A robust accumulation of GFP expressing marrow-derived cells were found in the vicinity of new blood vessel formation and inflammation (FITC 20x).

\section{SIS-ECM Scaffolds}

As with the autologous tendon repairs, no graft ruptures were observed at any time point. The SIS-ECM graft was still distinguishable 1 week after surgery, but by 2 weeks the SIS-ECM was incorporated into the remodeling tendon and could not be identified as a discrete entity. Similar to the autologous tendon repair group, the SISECM repair group showed a dense mononcuclear cell infiltration and a rich blood supply at 1 and 
A

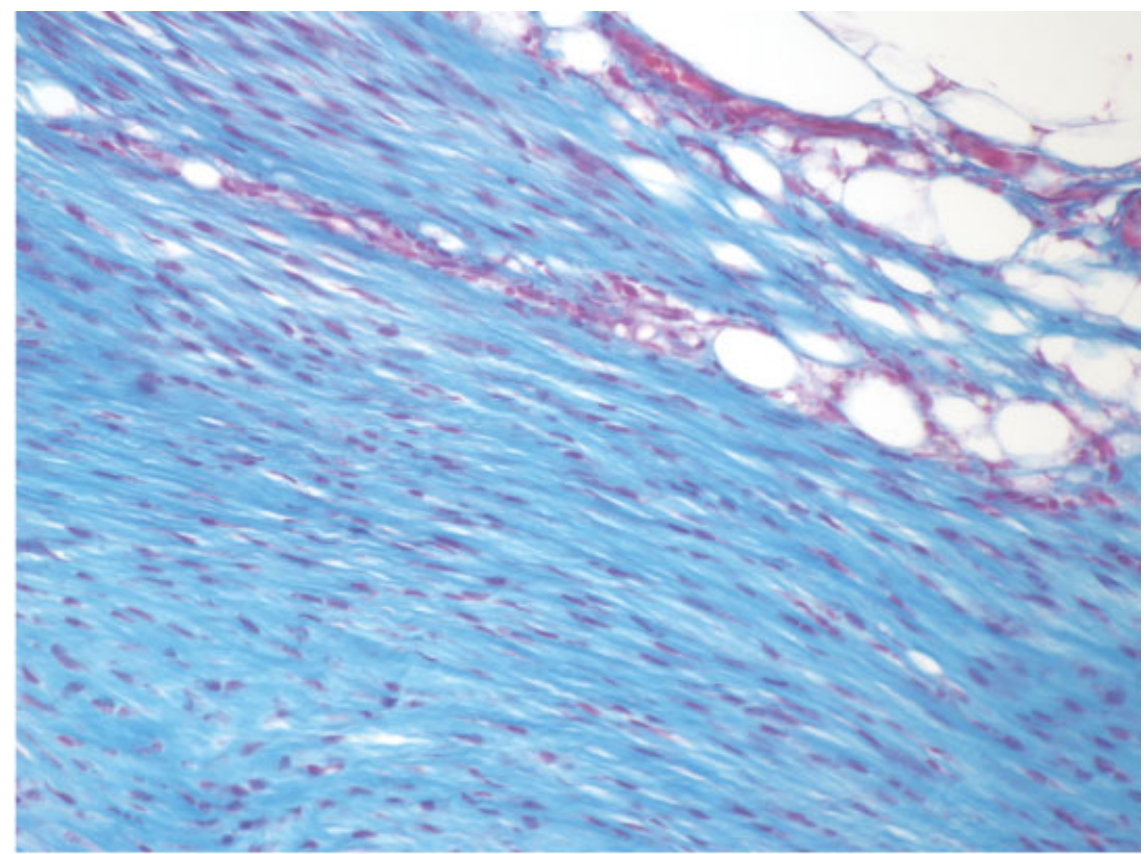

B

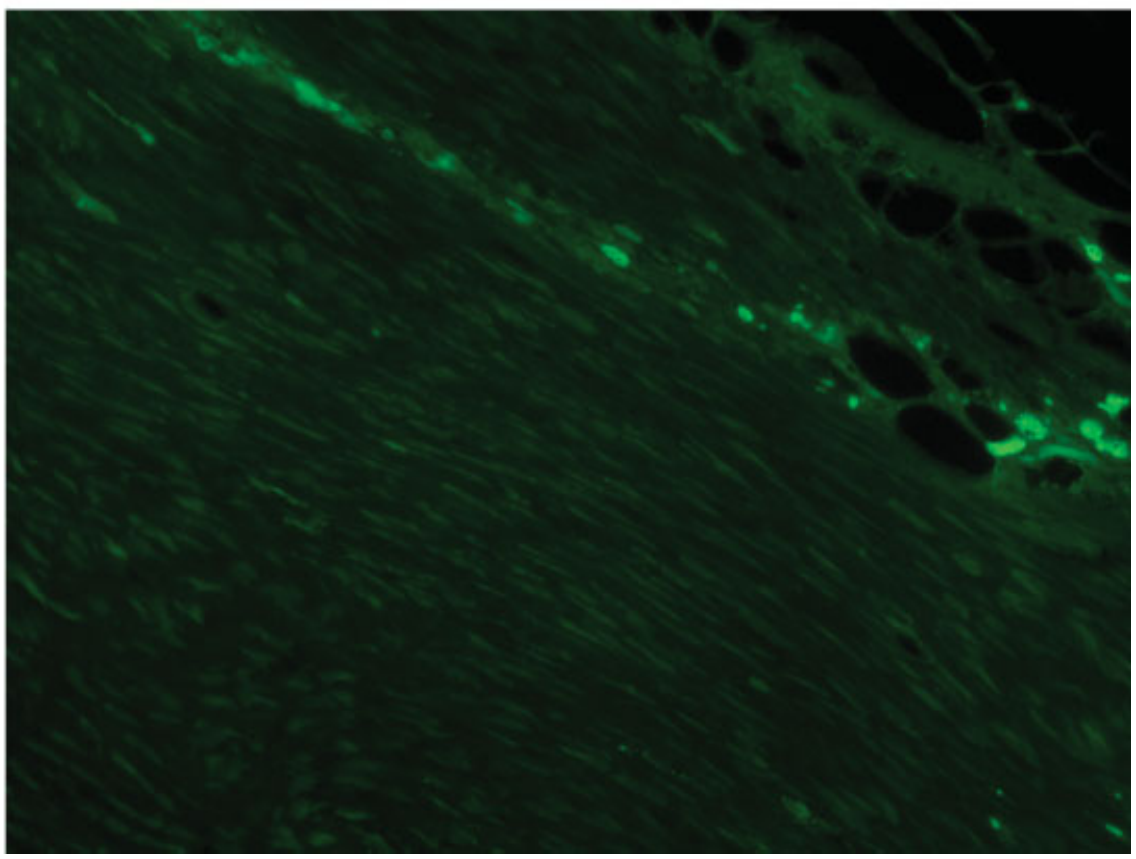

Figure 3. (A) At 4 weeks after surgery, the autologous tendon repair group showed organized collagenous tissue and a diminished cell infiltration compared with 2 weeks after surgery (Masson's Trichrome 20×). (B) GFP expressing marrow-derived cells were found only in the vicinity of new blood vessels or areas of increased cellularity (FITC 20×).

2 weeks after surgery. An abundant population of GFP expressing marrow-derived cells was observed in the remodeling tendon both in the vicinity of vascular structures and in the tendon body
(Fig. 5A and B). At 4 weeks postsurgery (Fig. 6A and $\mathrm{B}$ ), a dense mononuclear cell population persisted at the site of scaffold placement, and these cells consisted of both mononuclear round 


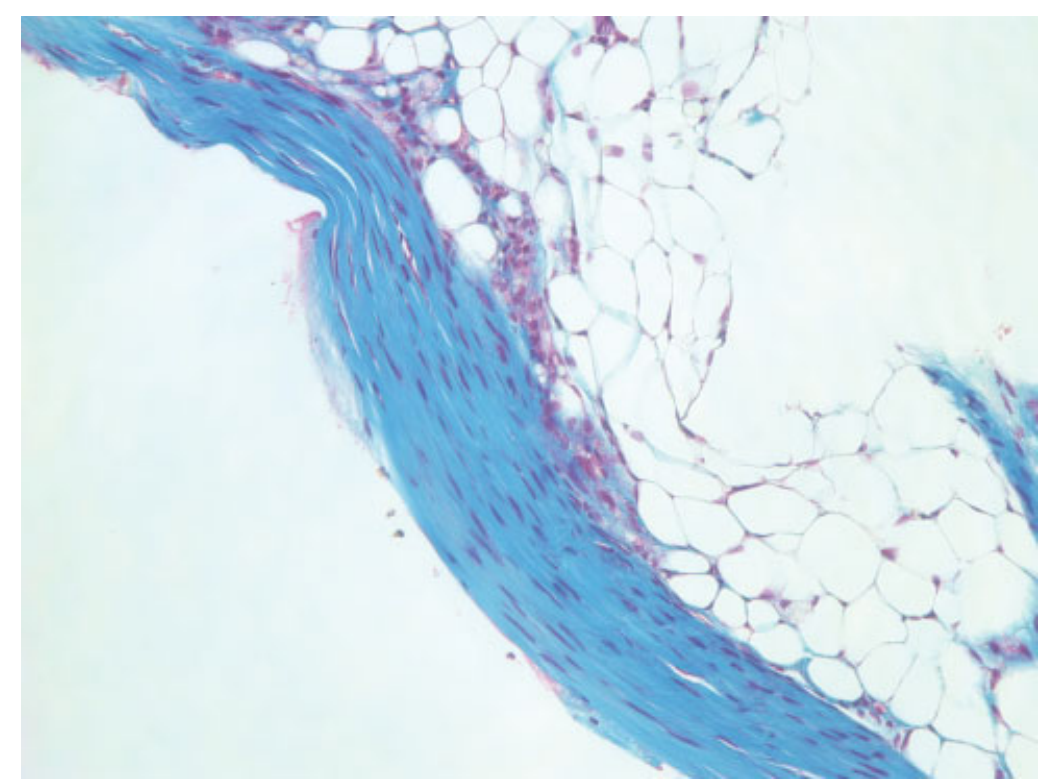

Figure 4. At 16 weeks after surgery, the autologous tendon repair group showed diminished cell infiltration to near normal, and the remodeled tendon was organized collagenous tissue. No GFP cells were observed in the tendon body (Masson's Trichrome 20×).

cells and spindle-shaped cells. There was no morphologically distinguishable SIS-ECM scaffold material remaining, and the suture lines between the native tissue and the healing tissue could not be identified. The SIS-ECM repair group showed more marrow-derived green fluorescent cells when compared to the autologous tendon repair group at 4 weeks. The cellularity of the SIS-ECM scaffold site had diminished by 8 weeks after surgery, but was still greater than would be found in normal tendon tissue. No obvious inflammatory response could be seen at the 8-week time point. At both 8 and 16 weeks, the remodeled tendon of the SIS-ECM group continued to show GFP positive marrow-derived cells distributed uniformly throughout the graft segment. The green fluorescent marrow-derived cells within the body of the tendon were typically spindleshaped and GFP-positive cells were also noted in the paratendinous tissue (Fig. 7).

\section{DISCUSSION}

This study confirms that SIS-ECM supports constructive remodeling of the Achilles tendon in a mouse model, and shows that a bone marrowderived cell population are involved in the longterm remodeling process. Although the role of these cells in the remodeling process is not yet

Table 1. Qualitative Prevalence of Inflammatory Cells, Bone Marrow-Derived Cells, Mononuclear Cells, and Collagenous Tissue in the Autologous Tendon Repair Group: (Control) and the SIS-ECM Group Compared to Normal Tendon

\begin{tabular}{|c|c|c|c|c|c|c|c|c|}
\hline \multirow[b]{2}{*}{ Weeks } & \multicolumn{2}{|c|}{ Inflammatory Cells } & \multicolumn{2}{|c|}{$\begin{array}{c}\text { Bone Marrow-Derived } \\
\text { Cells }\end{array}$} & \multicolumn{2}{|c|}{ Mononuclear Cells } & \multicolumn{2}{|c|}{$\begin{array}{l}\text { Collagenous } \\
\text { Tissue }\end{array}$} \\
\hline & Control & SIS & Control & SIS & Control & SIS & Control & SIS \\
\hline 1 & +++ & +++ & +++ & +++ & +++ & +++ & $\downarrow$ & $\downarrow \downarrow$ \\
\hline 2 & +++ & +++ & +++ & +++ & +++ & +++ & $\downarrow$ & $\downarrow$ \\
\hline 4 & ++ & + & + & ++ & ++ & ++ & - & - \\
\hline 8 & + & - & - & + & + & + & + & + \\
\hline 16 & - & - & - & + & + & + & + & + \\
\hline
\end{tabular}

+++ , robust increase; ++ , moderate increase; + , slight increase; - , normal level; $\downarrow$, slight decrease; $\downarrow \downarrow$, moderate decrease. 
A

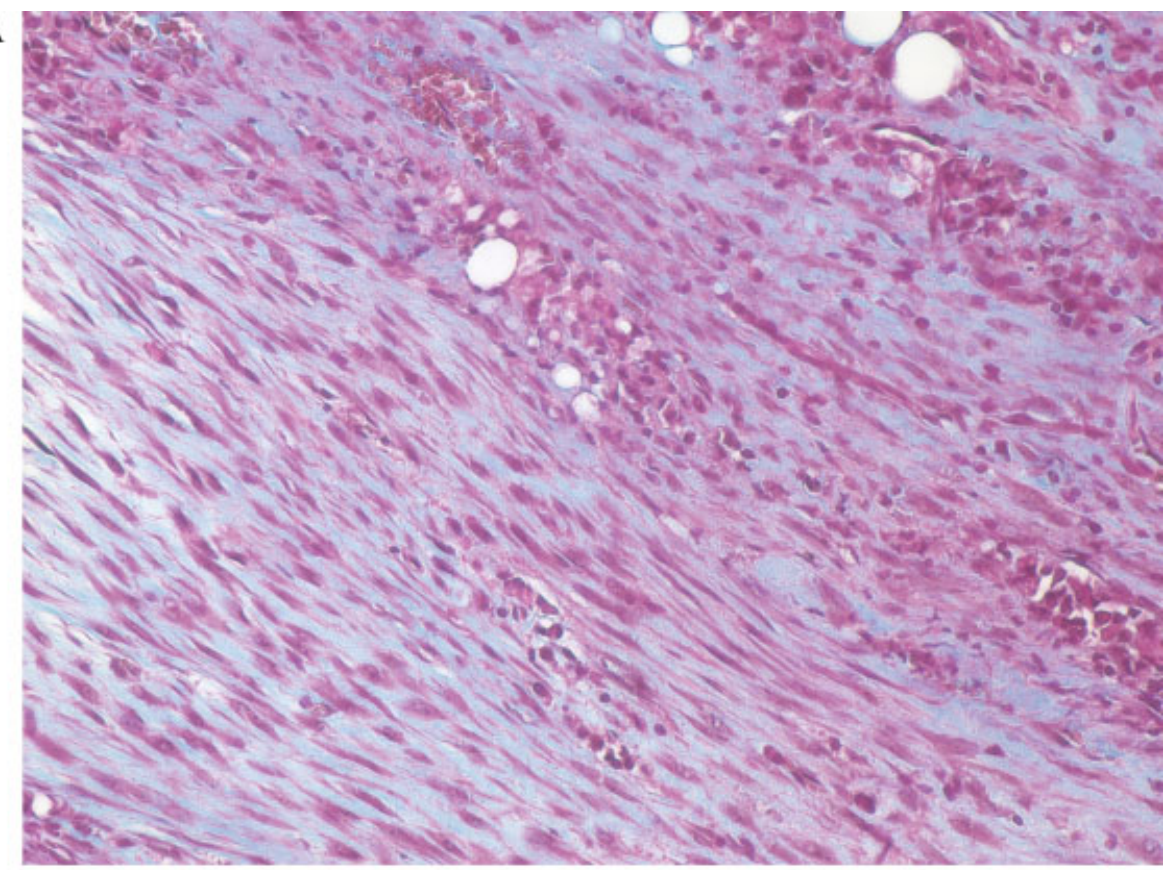

B

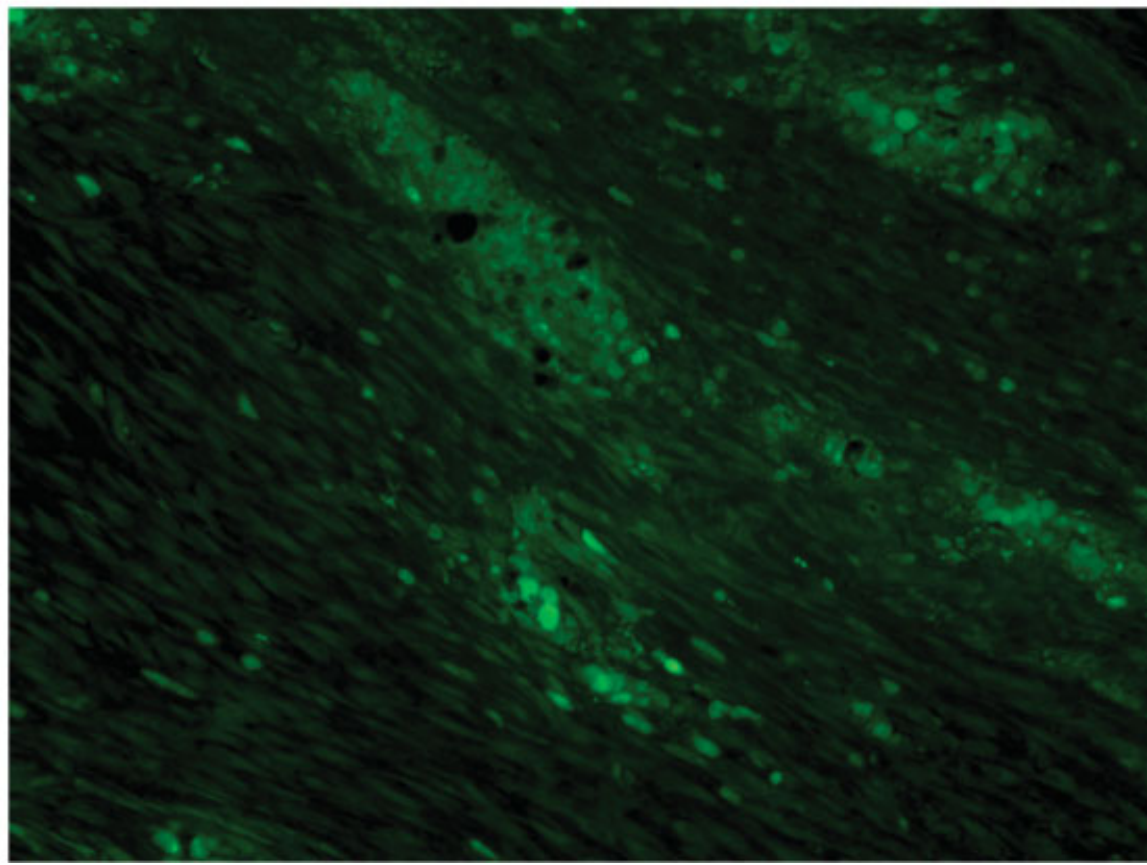

Figure 5. (A) At 2 weeks after surgery, the SIS-ECM repair group showed dense mononuclear cell infiltration, abundant blood vessels, and acute inflammation (Masson's Trichrome 20×). (B) There was also a robust accumulation of GFP expressing marrowderived cells in the vicinity of new blood vessel formation and inflammation, as well as in the tendon body (FITC $20 \times$ ).

fully understood, it has been observed that they remain beyond the inflammatory stage of healing and are found throughout the midsubstance of the tendon when healing is complete. These findings suggest that the bone marrow-derived cells that populate the SIS-ECM may represent a population of cells that can become site specific cells that reside in normal tendon tissue, such as fibroblasts and/or endothelial cells. In the absence of SISECM, no bone marrow-derived cells are found 
$\mathbf{A}$

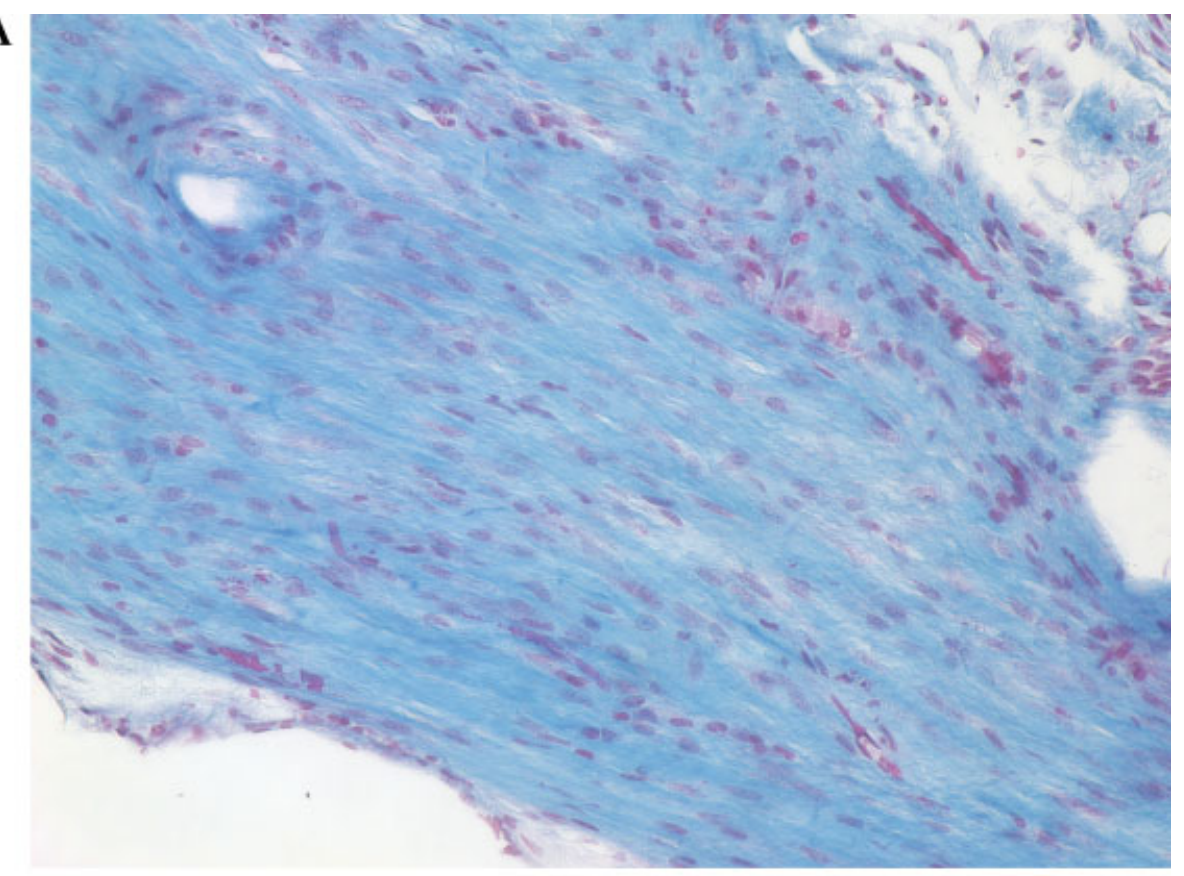

B

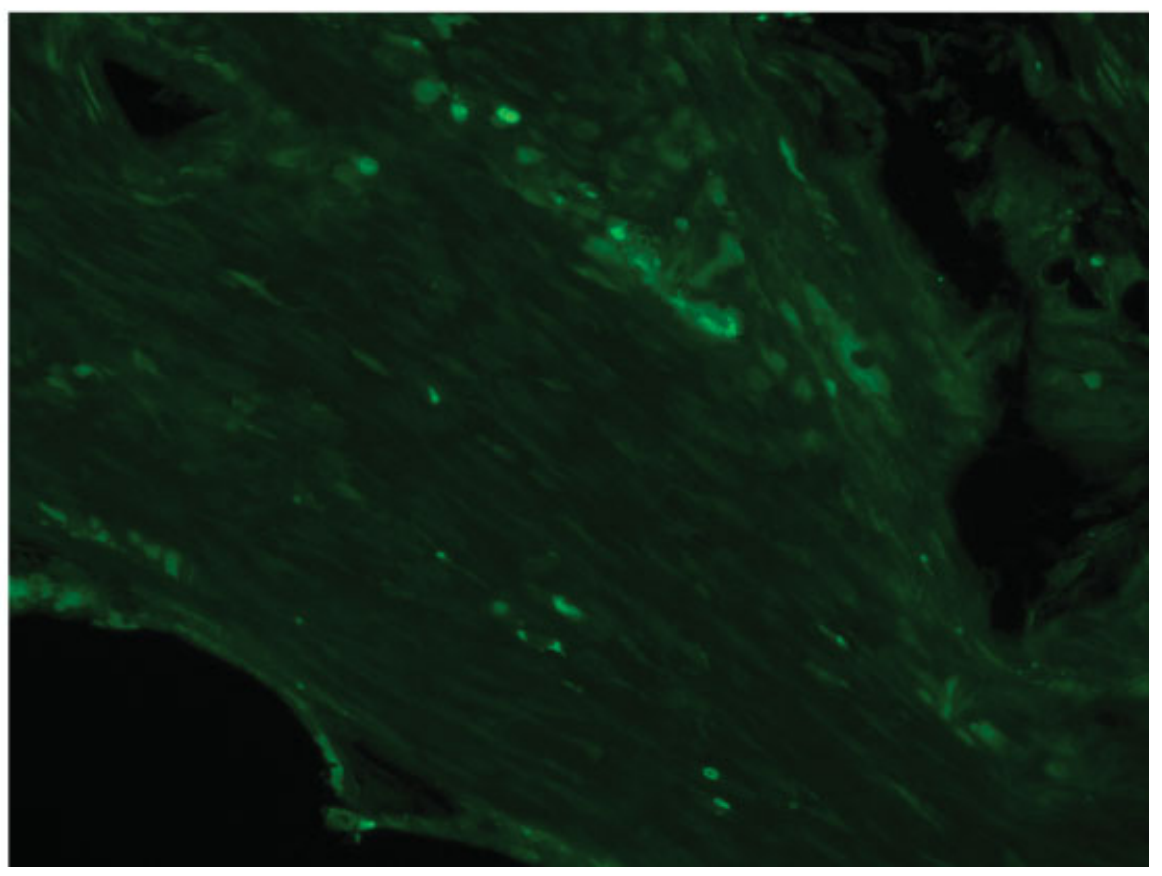

Figure 6. (A) At 4 weeks after surgery, the SIS-ECM repair group showed diminished cellular infiltration compared with 2 weeks after injury, but greater cellularity than normal tendon. The remodeling tendon is organized collagenous tissue (Masson's Trichrome 20×). (B) A robust accumulation of GFP expressing marrow-derived cells were found in the vicinity of new blood vessel formation and inflammation, as well as in the tendon body (FITC $20 \times$ ).

within the body of the remodeled tendon. In the current model, it was expected that the autologous tendon control would heal well because the tissue used in the repair was healthy, and the devitalized autologous tendon graft was replaced immediately after the defect was created. In the clinical scenario, the control group would more closely resemble an acute Achiles tendon injury 


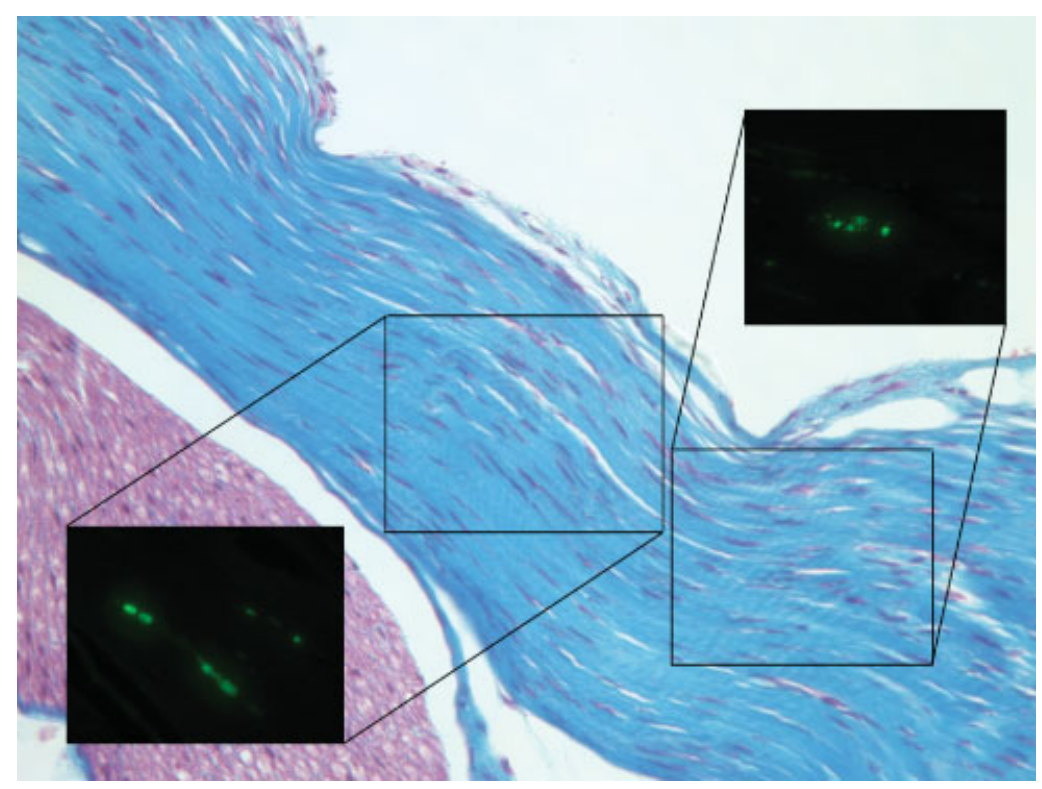

Figure 7. At 2 weeks after surgery, the SIS-ECM repair group showed that the cellularity had decreased to near normal, and the tendon consisted primarily of organized collagenous tissue with increased vascularity compared to normal tendon. The inlays show the GFP expressing marrow-derived cells present within organized collagenous tendon tissue (Masson's Trichrome 20×; insets FITC 20×).

and repair. A neglected Achilles tear with tendon retraction would require a method to "fill" the gap. In these cases of neglected repair and retraction, the presence of a bone marrow-derived cell with the potential to differentiate into a tissue-specific cell may have a beneficial effect on tissue healing and remodeling.

Marrow-derived mesenchymal progenitor cells have been identified to have the potential to differentiate into a variety of types of cells in vitro and in vivo. ${ }^{24,25}$ Their potential to improve healing is part of the rationale for autologous transplants of bone marrow aspirates for bone repair in the clinical setting. ${ }^{26,27}$ There is increasing evidence that marrow-derived mesenchymal progenitor cells can enter circulation and participate in remodeling of tissue during development and homeostasis, or following injury. ${ }^{28-31}$ A similar chimeric mouse model to that used in the present study showed that marrow-derived cells populated a number of organs, including the heart, lung, kidney, small intestine, and skin due to normal homeostatis. ${ }^{28}$ When an injury was created in the skin, it was observed that a population of nonhematopoietic bone marrow-derived cells were present in the wound site, were synthesizing collagen types I and III, and had a histological resemblence to fibroblasts. In another study, ${ }^{32}$ a portion of fetal mouse small intestine was trans- planted into the rectus abdominus of a chimeric mouse expressing GFP in all of its bone marrow cells. Nonhematopoietic marrow-derived cells were observed in the lamina propria of the implanted intestinal tissue. This same layer of the small intestine, that is, the lamina propria, is included in the SIS-ECM scaffold material. Similar findings were observed in both mice and humans in which female recipients received bone marrow from a male donor. ${ }^{33}$ Myofibroblasts positive for the $\mathrm{Y}$ chromome were observed in the lamina propria of the small intestine.

In the present study, no GFP expressing marrow-derived cells were observed within the native tendon or within the fully remodeled autologous tendon tissue graft. Occasional marrow-derived cells were observed in the vicinity of new blood vessels and in the paratendinous tissue of the Achilles tendon repaired with autologous tendon tissue. These findings suggest that the marrow-derived cells are not typically involved in the homeostasis or long-term remodeling after injury of the native Achilles tendon.

It has recently been shown in vitro that small peptides (5-16 kDa) formed during chemical and physical degradation of ECM bioscaffolds, including SIS-ECM, are chemoattractant for numerous cell types, including endothelial cells. ${ }^{34} \mathrm{~A}$ similar chemoattractant mechanism may also be responsible for 
the recruitment of marrow-derived mesenchymal progenitor cells to the site of the ECM remodeling. We speculate that the constructive remodeling process associated with naturally dervived ECM scaffolds is due at least in part to the participation of autogenous circulating marrow-derived progenitor cells that are recruited by the degradation products of the SIS-ECM. It is plausible that such cells would then differentiate in response to local environmental cues, and thus contribute to tissue organization and differentiation. It has also been shown that degradation products of SIS-ECM have an antimicrobial effect, ${ }^{35-38}$ and that without ECM degradation this effect is not observed. ${ }^{39}$ Factors that inhibit the ECM degradation process, such as chemical crosslinking, may interfere with the biological activities that have been demonstrated by the products of ECM degradation.

The specific phenotype of the GFP expressing marrow-derived cells involved in remodeling of the ECM graft was not determined in the present study, nor have the specific components of the ECM that attract the marrow-derived cells to the site of healing been identified. Despite these limitations, the results of this study show that a population of marrow-derived cells participates in the long-term remodeling of a degradable ECM scaffold when used as a repair device in this murine model of Achilles tendon repair. Such cells do not contribute to tendon healing in the absence of the ECM biologic scaffold. The translation of these findings to the tendon healing process in humans remains to be shown.

\section{ACKNOWLEDGMENTS}

The authors acknowledge our funding sources, NIH\# EB000261 and DePuy Orthopaedics.

\section{REFERENCES}

1. Lee MS. 2004. GraftJacket augmentation of chronic Achilles tendon ruptures. Orthopedics 27:s151s153.

2. Metcalf MH, Savoie FH, Kellum B. 2002. Surgical technique for xenograft (SIS) augmentation of rotator-cuff repairs. Oper Tech Orthop 12:204208.

3. Sclamberg SG, Tibone JE, Itamura JM, et al. 2004. Six-month magnetic resonance imaging follow-up of large and massive rotator cuff repairs reinforced with porcine small intestinal submucosa. J Shoulder Elbow Surg 13:538-541.
4. Badylak S, Kokini K, Tullius B, et al. 2002. Morphologic study of small intestinal submucosa as a body wall repair device. J Surg Res 103:190-202.

5. Badylak S, Obermiller J, Geddes L, et al. 2003. Extracellular matrix for myocardial repair. Heart Surg Forum 6:E20-E26.

6. Badylak SF, Tullius R, Kokini K, et al. 1995. The use of xenogeneic small intestinal submucosa as a biomaterial for Achilles tendon repair in a dog model. J Biomed Mater Res 29:977-985.

7. Cobb MA, Badylak SF, Janas W, et al. 1996. Histology after dural grafting with small intestinal submucosa. Surg Neurol 46:389-393; discussion 393-394.

8. Dejardin LM, Arnoczky SP, Ewers BJ, et al. 2001. Tissue-engineered rotator cuff tendon using porcine small intestine submucosa. Histologic and mechanical evaluation in dogs. Am J Sports Med 29:175-184.

9. Hodde JP, Badylak SF, Shelbourne KD. 1997. The effect of range of motion on remodeling of small intestinal submucosa (SIS) when used as an Achilles tendon repair material in the rabbit. Tissue Eng 3:27-37.

10. Kropp BP, Ludlow JK, Spicer D, et al. 1998. Rabbit urethral regeneration using small intestinal submucosa onlay grafts. Urology 52:138-142.

11. Musahl V, Abramowitch SD, Gilbert TW, et al. 2004. The use of porcine small intestinal submucosa to enhance the healing of the medial collateral ligament-a functional tissue engineering study in rabbits. J Orthop Res 22:214-220.

12. Record RD, Hillegonds D, Simmons C, et al. 2001. In vivo degradation of $14 \mathrm{C}$-labeled small intestinal submucosa (SIS) when used for urinary bladder repair. Biomaterials 22:2653-2659.

13. Hodde JP, Record RD, Liang HA, et al. 2001. Vascular endothelial growth factor in porcinederived extracellular matrix. Endothelium 8:11-24.

14. Voytik-Harbin SL, Brightman AO, Kraine MR, et al. 1997. Identification of extractable growth factors from small intestinal submucosa. J Cell Biochem 67:478-491.

15. Hodde JP, Ernst DM, Hiles MC. 2005. An investigation of the long-term bioactivity of endogenous growth factor in OASIS Wound Matrix. J Wound Care 14:23-25.

16. McDevitt CA, Wildey GM, Cutrone RM. 2003. Transforming growth factor-beta1 in a sterilized tissue derived from the pig small intestine submucosa. J Biomed Mater Res A 67:637-640.

17. Arner O, Lindholm A, Orell SR. 1959. Histologic changes in subcutaneous rupture of the Achilles tendon: a study of 74 cases. Acta Chir Scand 116: 484-490.

18. Butler DL, Juncosa N, Dressler MR. 2004. Functional efficacy of tendon repair processes. Annu Rev Biomed Eng 6:303-329. 
19. Enwemeka CS. 1989. Inflammation, cellularity, and fibrillogenesis in regenerating tendon: implications for tendon rehabilitation. Phys Ther 69:816825.

20. Badylak SF, Park K, Peppas N, et al. 2001. Marrow-derived cells populate scaffolds composed of xenogeneic extracellular matrix. Exp Hematol 29:1310-1318.

21. Freytes DO, Badylak SF, Webster TJ, et al. 2004. Biaxial strength of multilaminated extracellular matrix scaffolds. Biomaterials 25:2353-2361.

22. Sacks MS, Gloeckner DC. 1999. Quantification of the fiber architecture and biaxial mechanical behavior of porcine intestinal submucosa. J Biomed Mater Res 46:1-10.

23. Yoder MC, Cumming JG, Hiatt K, et al. 1996. A novel method of myeloablation to enhance engraftment of adult bone marrow cells in newborn mice. Biol Blood Marrow Transplant 2:59-67.

24. Caplan AI. 1991. Mesenchymal stem cells. J Orthop Res 9:641-650.

25. Prockop DJ. 1997. Marrow stromal cells as stem cells for nonhematopoietic tissues. Science 276:7174.

26. Lokiec F, Ezra E, Khermosh O, et al. 1996. Simple bone cysts treated by percutaneous autologous marrow grafting. A preliminary report. J Bone Joint Surg Br 78:934-937.

27. Wientroub S, Goodwin D, Khermosh O, et al. 1989. The clinical use of autologous marrow to improve osteogenic potential of bone grafts in pediatric orthopedics. J Pediatr Orthop 9:186-190.

28. Fathke C, Wilson L, Hutter J, et al. 2004. Contribution of bone marrow-derived cells to skin: collagen deposition and wound repair. Stem Cells 22:812822.

29. Krause DS, Theise ND, Collector MI, et al. 2001. Multi-organ, multi-lineage engraftment by a single bone marrow-derived stem cell. Cell 105:369-377.

30. Liechty KW, MacKenzie TC, Shaaban AF, et al. 2000. Human mesenchymal stem cells engraft and demonstrate site-specific differentiation after in utero transplantation in sheep. Nat Med 6:12821286.

31. Tepper OM, Capla JM, Galiano RD, et al. 2005. Adult vasculogenesis occurs through in situ recruitment, proliferation, and tubulization of circulating bone marrow-derived cells. Blood 105:1068-1077.

32. Kato Y, Yamataka A, Miyahara K, et al. 2004. Recipient non-hematopoietic bone marrow cells in the intestinal graft after fetal small intestinal transplantation. Pediatr Surg Int 20:1-4.

33. Brittan M, Hunt T, Jeffery R, et al. 2002. Bone marrow derivation of pericryptal myofibroblasts in the mouse and human small intestine and colon. Gut 50:752-757.

34. Li F, Li W, Johnson S, et al. 2004. Low-molecularweight peptides derived from extracellular matrix as chemoattractants for primary endothelial cells. Endothelium 11:199-206.

35. Badylak SF, Coffey AC, Lantz GC, et al. 1994. Comparison of the resistance to infection of intestinal submucosa arterial autografts versus polytetrafluoroethylene arterial prostheses in a dog model. J Vasc Surg 19:465-472.

36. Badylak SF, Wu CC, Bible M, et al. 2003. Host protection against deliberate bacterial contamination of an extracellular matrix bioscaffold versus Dacron mesh in a dog model of orthopedic soft tissue repair. J Biomed Mater Res B Appl Biomater 67:648-654.

37. Jernigan TW, Croce MA, Cagiannos C, et al. 2004. Small intestinal submucosa for vascular reconstruction in the presence of gastrointestinal contamination. Ann Surg 239:733-738; discussion 738-740.

38. Sarikaya A, Record R, Wu CC, et al. 2002. Antimicrobial activity associated with extracellular matrices. Tissue Eng 8:63-71.

39. Holtom PD, Shinar Z, Benna J, et al. 2004. Porcine small intestine submucosa does not show antimicrobial properties. Clin Orthop 427:18-21. 